[Presentación del Número]

Suscribirse a la revista

http://dx.doi.org/10.22402/j.rdipycs.unam.1.2.2015.19.185-192

\title{
Presentación del Número de la Revista
}

| Volumen 1 | Número 2 | Diciembre 2015 |

\section{TABLA DE CONTENIDO}

INTRODUCCIÓN

ESQUEMA EDITORIAL

ANÁLISIS DE LOS DICTÁMENES DE LOS REVISORES

Perfil de evaluación entre pares, 187

Introducción, 187

Método, 187

Resultados , 188

Todos los apartados, 188

Calificación Promedio de los artículos, 188

Índice de concordancia, 189

Correlograma, 189

Deacuerdo entre revisores, 189

El MATERIAL CIENTÍfICO PUBLICADO

190

REFERENCIAS

191 


\section{INTRODUCCIÓN}

$\mathrm{E}$ ste segundo numero de 2015 de la Revista Digital Internacional de Psicología y Ciencia Social (RDIPyCS) representa un cumulo de esfuerzos para el equipo editorial que involucraron por un lado, el rediseño de la revista para que se ajustará mejor a los parámetros de los motores de búsqueda de Internet; y por otro, la automatización de todo el proceso editorial a través del Open Journal Sytem (OJS), puesto que históricamente en la revista se había utilizado esta herramienta, prácticamente sólo como repositorio de artículos.

Estos esfuerzos tomaron como punto de referencia las mejores prácticas mundiales en la edición de revistas científicas, en consecuencia, nuestra revista se publica ahora en diferentes formatos; en el clásico PDF, en el dinámico e interactivo HTML, en Epub y en mp3.

El formato Epub permitirá que nuestra revista pueda ser leída a través de múltiples dispositivos como serían tablets, móviles, videoconsolas, lectores de libros electrónicos, etc. De igual manera, los documentos publicados podrán adaptarse a distintos tamaños de letra y pantalla, además nuestros lectores contarán con herramientas que les permitirán marcar los pasajes de los materiales publicados que les parezcan más interesantes y compartirlos con sus colegas o pares.

Finalmente, siguiendo una política editorial de inclusión social los artículos científicos se publicarán también en formato mp3, con la finalidad de que sirvan como herramientas de apoyo para personas débiles visuales o ciegas.

\section{ESQUEMA EDITORIAL}

Continuando con su vocación de transparentar las decisiones científico-editoriales, este número de la RDIPyCS muestra al mundo todos los procesos que utiliza para transferir y difundir la ciencia.

Estos procesos se desarrollarán en un esquema editorial que será usado en todos los artículos que se publiquen y consiste en:

1. Portada. Está constituida por título, el resumen en español e inglés de artículo, las palabras claves y la forma de citar de acuerdo a las reglas establecidas por el American Psychological Association (APA).

2. Bitácora. Conformada por el título del artículo, los tiempos editoriales (cuándo se recibió, cuándo fue aceptado para su publicación y la fecha en que fue publicado); las imágenes iconográficas de los dos primeros autores/as del artículo, en caso de que sean más; las secciones de resúmenes curriculares; las contribuciones que hicieron cada uno de los autores/as en la elaboración del artículo; los agradecimientos y los datos de afiliación de las autores/as.

3. Índice. Es una serie de vínculos que permitirán al lector navegar en forma libre por el cuerpo del artículo, lo que evitará una lectura secuencial del material científico publicado.

4. Cuerpo. Consiste en el artículo propiamente. Los apartados en que se desarrolle el material científico dependerán del tipo de estudio que se esté reportando. Si desea conocer los que se aceptan en esta revista diríjase al siguiente vínculo.

5. Meta-análisis del artículo. Está constituido por procedimientos meta-analíticos en donde se presentan cuantitativamente las opiniones que emitieron los evaluadores sobre el artículo. Esta sección del artículo tiene dos dimensiones: la primera es la cuantitativa, constituida por tres partes, (i) el perfil en donde se presentan las opiniones que emitieron los revisores en cuanto a la calidad de la introducción, la metodología, los resultados y todos los apartados del artículo. (ii) La segunda parte presenta la figura de concordancia que existió entre las opiniones de los revisores, construida utilizando el método de Bland y Altman (1986). Finalmente, (iii) la tercera muestra el nivel de acuerdo que se dio entre los revisores al calificar todos los apartados del material científico. La otra dimensión es la cualitativa del meta-análisis en donde se presentan los juicios emitidos por los revisores sobre el manuscrito científico evaluado.

6. Historia del proceso editorial. En él hay dos vínculos, uno permite el acceso al meta-análisis en línea, y en el otro se puede consultar todo el proceso editorial del artículo, que incluye las conversaciones entre los autores y los revisores, es decir, el intercambio dialógico entre los actores científicos.

\section{ANÁlisis de LOS DICTÁMENES de lOS REVISORES}

En esta sección se presenta un análisis global de los dictámenes que los revisores hicieron de todos los artículos publicados en este número de la revista. Podría decirse que es una especie de radiografía en conjunto que nos 
habla de la opinión que los revisores se formaron de los materiales científicos publicados.

Con esto se busca que el lector tome como punto de referencia la opinión de los revisores, que son gente de la comunidad científica; la contraste con su opinión y saque sus propias conclusiones sobre la calidad, relevancia e impacto de lo que se publicó en este número.

De esta manera se irá formando una opinión pública fundamentada en la transparencia de las decisiones que se toman para transmitir el conocimiento científico; es decir, una opinión pública informada producto del entendimiento personal que tiene cada revisor/a y de la valoración que hagan los lectores del material científico divulgado.

Al igual que en cada artículo, en este apartado de la revista se presenta el meta-análisis, pero a diferencia de aquel, aquí se muestra el resultado de la opinión que emitieron los revisores/as en su conjunto de los artículos publicados. Las secciones son: el perfil de evaluación entre pares, la calificación promedio otorgada a cada uno de los artículos, el índice de concordancia, el correlograma y el índice de desacuerdo entre revisores/as.

Es importante mencionar que aunque se habla de los revisores/as, esto no significa que cada uno de ellos haya evaluado todos los artículos publicados en la revista, sino más bien para este meta-análisis se consideró a cada revisor como una unidad de análisis y cada artículo como si fuera uno mismo, es decir, el conjunto de dictámenes se tomó a manera de una unidad replicativa, ya que cada dictamen fue hecho por diferentes revisores/as.

Si bien como lo señalan Glass, Wilson, y Gottman (1975); Yin (1984); Menard (1991) y Silva-Rodríguez (2004), los análisis que se basan en unidades replicativas son más propensos a verse afectados por fuentes de invalidez, debido a la alteración de la naturaleza de los individuos (en nuestro caso diferentes revisores/as) que componen la unidad de observación, puesto que es probable confundir el efecto de los juicios de los revisores/ as con cambios abruptos en la composición de dicha unidad. Sin embargo, la unidad replicativa está menos sujeta a efectos de información y reactividad, puesto que si fueran los mismos revisores/es de todos los artículos que se publican en un determinado número de la revista acarrearían su sesgo en todos los artículos evaluados.

Tomando en consideración lo anterior, el algoritmo que se utilizó para calcular el perfil de la revista, los índices de concordancia y acuerdo, así como el correlograma y la calificación otorgada a los artículos de este número fue el siguiente:

$$
\text { Opinión }=\frac{\left(a_{11}+a_{21}\right)+\left(a_{32}+a_{42}\right)+\ldots+\left(a_{i j}+a_{1 j}\right)}{n}
$$

En donde:

$a$ es la opinión de la revisión i del artículo j iésima revisión $=1,2, \ldots$, hasta $\boldsymbol{n}$, y

jésimo artículo $=1,2, \ldots$, hasta $\boldsymbol{k}$

Que para el caso de este número de la revista $\boldsymbol{n}$ es igual a 8 revisores y $\boldsymbol{k}$ igual a 4 artículos.

\section{Perfil de evaluación entre pares}

Por su parte el perfil evalúa la apreciación que le asignaron los revisores a las partes más amplias que constituyen el reporte de la literatura científica como son la introducción, el método, los resultados y todos los apartados del artículo.

\section{Introducción}

En la figura 1 se muestra la opinión que emitieron respecto a la introducción de todos los artículos. Como se puede observar en esa figura, los revisores opinaron que la definición de variables fue satisfactoriamente baja; debido a que hubieron algunos detalles que se omitieron, por lo que le asignaron la calificación de 2.05 de una calificación máxima de 4.

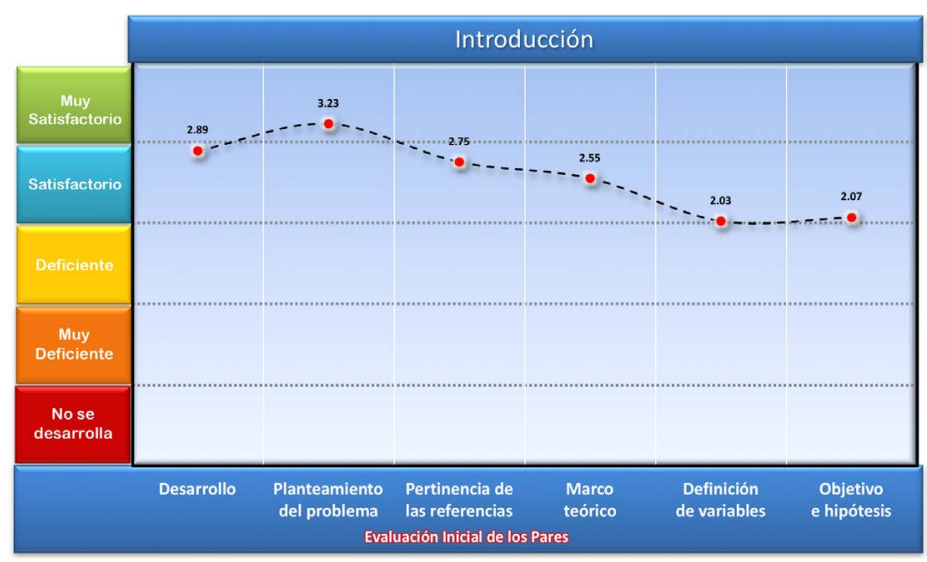

Figura 1.

Perfil de la opinión de los revisores sobre cada uno de los componentes de la introducción de los artículos.

Por otro lado, la opinión más favorable fue en el planteamiento del problema al conceder una puntuación de 3.23 de una de 4. Finalmente, al observar a lo largo de todo el patrón de opinión, los revisores consideraron que los artículos en cuanto a introducción eran muy satisfactorios, ya que el desarrollo, el planteamiento del problema, la pertinencia de las referencias, el marco teórico y el objetivo e hipótesis fueron calificados dentro de esa categoría. 


\section{Método}

Según los revisores, los artículos muestran una variabilidad considerable en el método, como se observa en la figura 2. Ellos consideraron que el desarrollo de las cuestiones éticas relacionadas con las temáticas abordadas por los artículos fue deficiente, puesto que la calificación que le otorgaron fue apenas de 1 de 4.

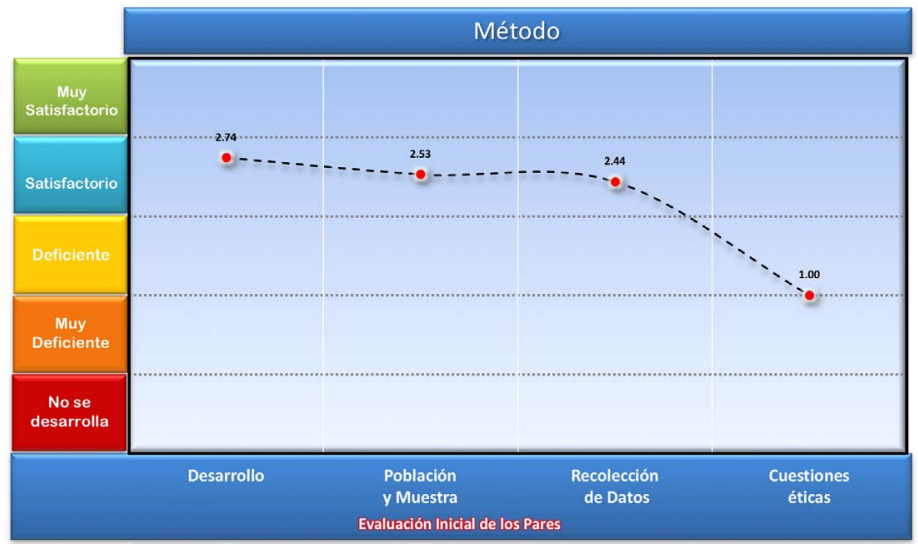

Figura 2.

Perfil de la opinión de los revisores sobre cada uno de los componentes del método de los artículos.

La opinión más favorable fue para los aspectos relacionados con la recolección de la información de los estudios, así como para la forma en que se desarrolló, se identificó a la población y se seleccionó la muestra, ya que todas éstas fueron consideradas como satisfactorias.

\section{Resultados}

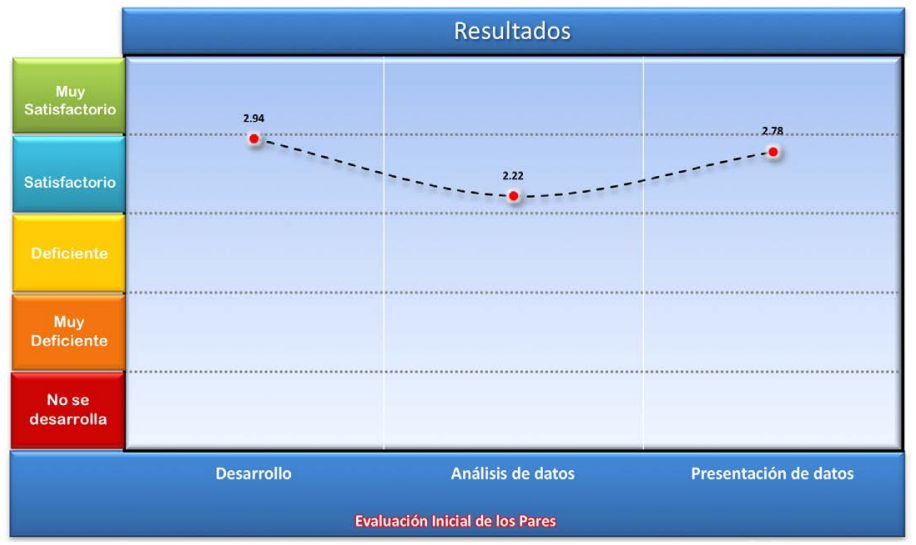

Figura 3.

Perfil de la opinión de los revisores sobre cada uno de los componentes de los resultados de los artículos.

En cuanto a la presentación de resultados (véase figura 3) las opiniones fueron más estables, ya que los revisores consideraron que la forma en que se desarrolló este apartado fue satisfactoria con una calificación de 2.94 de 4. Además consideraron que el análisis de datos Ilevado a cabo en los artículos fue satisfactoria.

Finalmente la presentación de los datos mediante tablas y gráficas fue considerada satisfactoria, alcanzando una calificación de 2.78 .

\section{Todos los apartados}

En la figura 4 se muestra la opinión emitida sobre todos los apartados. Como se puede ver en esa figura, los revisores le otorgaron a las conclusiones una calificación de 2.33, que las colocan en una calidad de satisfactorias en todos los artículos que se publican en este número.

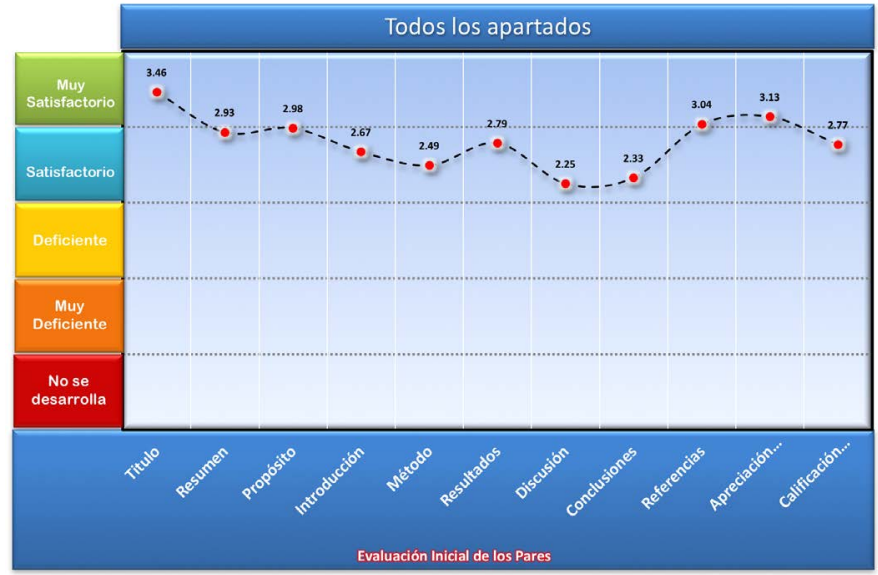

Figura 4.

Perfil de la opinión de los revisores en todos los apartados de los artículos.

Los otros apartados, como son el título, la referencias y sus apreciaciones, los consideraron muy satisfactorios; mientras que el resumen, propósito, introducción, método, resultados y la discusión las consideraron satisfactorias.

Por último, la calificación promedio otorgada a los artículos fue de 2.72, con lo cual puede decirse que los revisores opinaron que su calidad fue satisfactoria, ya que su apreciación cae levemente en esa categoría de la escala.

\section{Calificación Promedio de los artículos}

En la figura 5 se presenta la calificación promedio otorgada por los evaluadores al artículo que revisaron. En dicha figura puede observarse que la calificación otorgada a los artículos varió entre 88.39 y 57.31 , en una escala del 0 al 100, que corresponde al artículo escrito por la autora Ramos Ramírez y al escrito por Fabila y Hurtado, respectivamente.

Como se puede ver, la mayoría de los artículos obtuvieron una calificación variada el de Remigio y Pérez con un 59.19; y el de Valdez Jiménez y Villa con 71.97. 


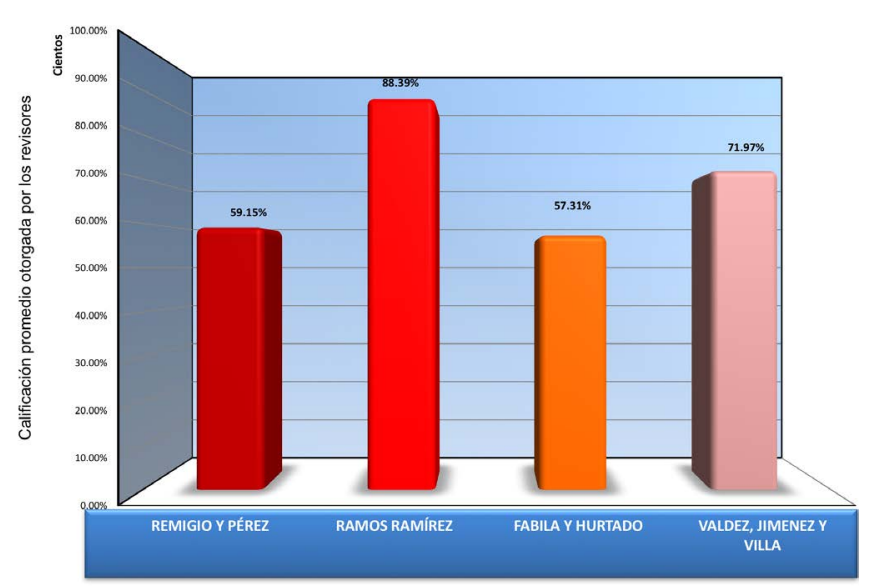

Figura 5.

Calificación promedio otorgada por los revisores al artículo.

\section{Índice de concordancia}

Este índice indica el acercamiento que hubo en los juicios de los revisores; es decir, la magnitud en que éstos reportan resultados iguales; mide por lo tanto la variabilidad entre sus opiniones o juicios con respecto a los dictámenes que emitieron del artículo.

Para construir la figura 6, se tomó la calificación promedio de los juicios que los revisores asignaron a las nueve partes del artículo, como fueron título, resumen, propósito, introducción, método, resultados, discusión, conclusiones y referencias.

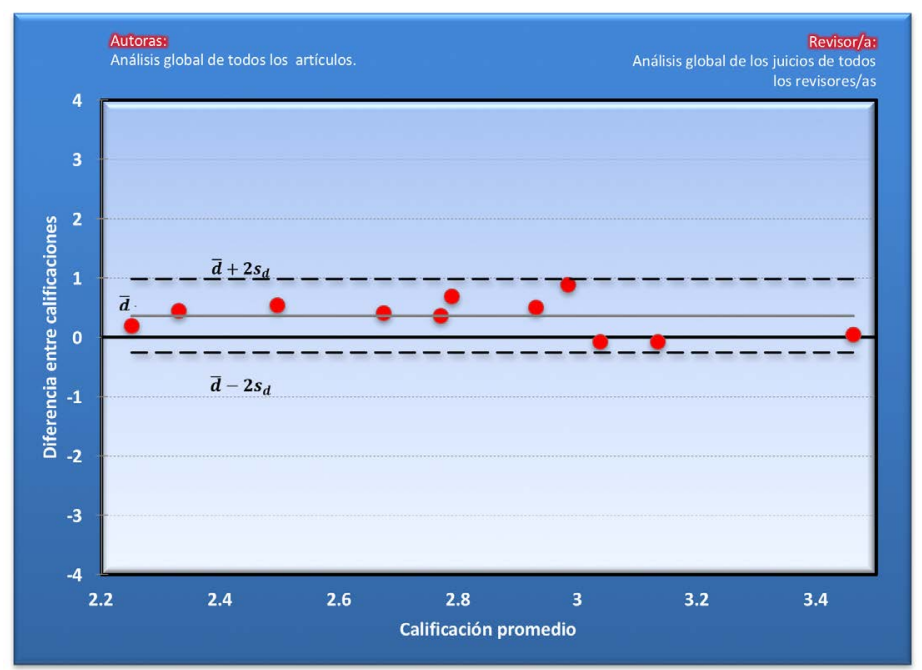

\section{Figura 6.}

Indice de concordancia en los juicios de los revisores.

En dicha figura se observa que la concordancia entre los revisores fue grande, ya que la diferencia entre ellos no superó el límite de las desviaciones estándar de las diferencias; solamente en el apartado de referencias la diferencia estuvo a punto de superar ese límite al obte- nerse una diferencia de 0.95 cuando el límite superior fue de 1.00 .

\section{Correlograma}

En la figura 7 se muestra el correlograma de los juicios que emitieron los revisores, es una figura construida a partir de calcular los índices de correlación entre las opiniones emitidas.

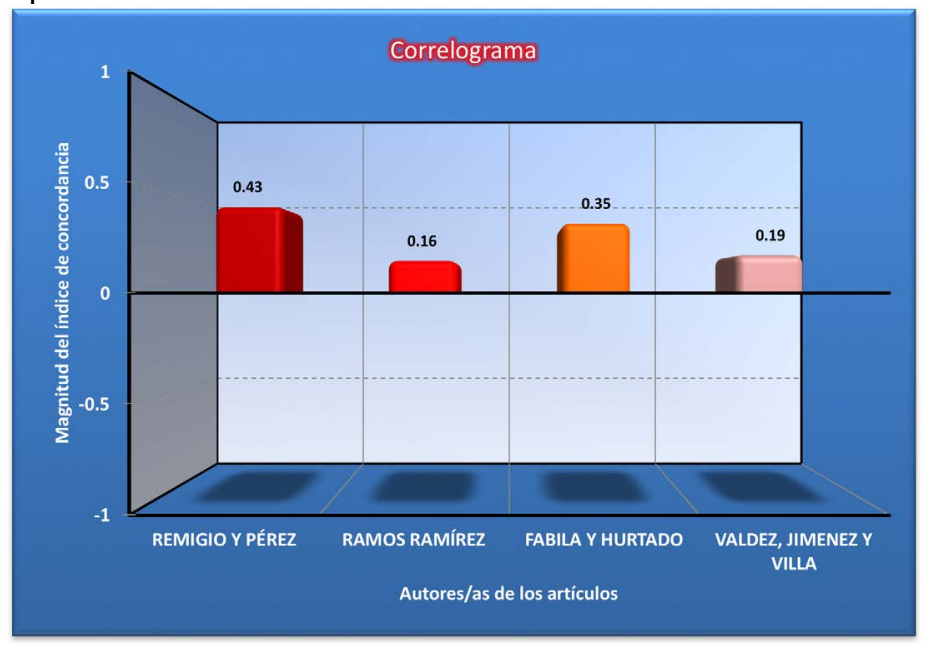

Figura 7.

Correlación entre los juicios de revisores.

Los juicios de los revisores que se correlacionaron en el mismo sentido y con una magnitud moderada fueron los que revisaron el artículo de Remigio y Pérez con un índice de 0.43 , después el de Fabila y Hurtado, con 0.35.

Las opiniones de los revisores que no se correlacionaron fueron los que dictaminaron el artículo de Ramos con 0.16, igualmente los que revisaron el artículo de Valdez, Jiménez y Villa sus juicios estuvieron contrapuesto debido a la magnitud de la correlación también fue baja, de 0.19, 3 décimas que el de Ramos.

\section{Desacuerdo entre revisores}

Finalmente la figura 8 muestra el desacuerdo entre los revisores, independientemente de su sentido, puesto que sólo evalúa la magnitud de la diferencia de opinión.

Los revisores que menos desacuerdos tuvieron fueron los que dictaminaron el artículo de Fabila y Hurtado, al otorgarle uno de ellos una calificación de 57.42 y el otro de 57.20.

Por otro lado, los desacuerdos más grandes en la opinión de los revisores se encontraron en los que dictaminaron el artículo de Váldez, Jiménez y Villa que fue de 32.74 puntos porcentuales, uno le otorgó la calificación de 88.44 mientras que el otro le designó un valor de 55.50.

También se observó una variabilidad muy grande en las opiniones de quienes dictaminaron el artículo de Remigio y Pérez, en donde la diferencia de la calificación entre 
ellos fue de 20.29 puntos porcentuales, uno le asignó un valor de 49.01 y el otro de 69.30. Igualmente la diferencia de opinión fue grande entre los revisores del artículo de Ramos, la cual alcanzo la magnitud de 17.22 puntos porcentuales, uno le asignó 97 puntos y el otro 79.78 .

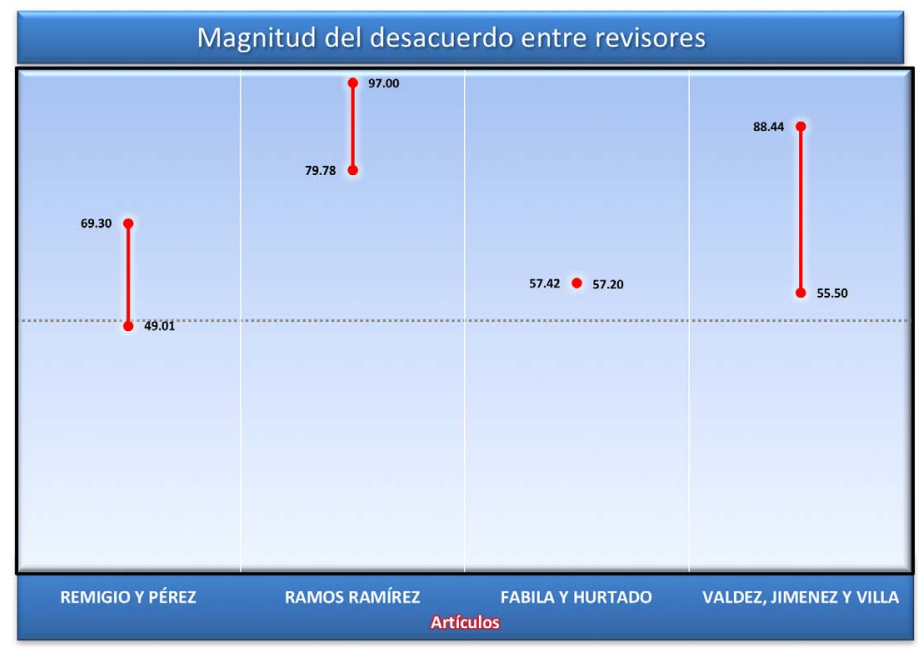

Figura 8.

Desacuerdo entre revisores.

Como se podría esperar por estos resultados la diferencia de opinión entre los revisores fue la regla más que la excepción. Esto confirma que los criterios utilizados son muy diversos y que no coinciden en sus apreciaciones sobre la calidad de un artículo.

Estas discrepancias en la apreciación de la calidad de los artículos son valoraciones que los revisores las perciben subjetivamente, lo relevante de todo esto es encontrar el punto en donde las diversas subjetividades, principalmente de los revisores, coincidan para así tener una revista que facilite la comunicación científica, no obstante que sea una realidad artificial producto de los múltiples significados y sentidos que los revisores le asignen al material científico revisado.

\section{El material CIENTífico PUBLICAdO}

En el mundo científico se está viviendo en esta época posmoderna cambios vertiginosos que han producido rupturas epistemológicas que tienen que ver con la racionalidad que se sigue para realizar la investigación científica. En este entorno de cambio, las revistas que difunden el quehacer científico necesitan alinear sus políticas editoriales para convertirse en un factor de cambio hacia una nueva forma de hacer y validar el conocimiento que generen las comunidades de científicos.

Es innegable que cada día que pasa se están interrelacionando distintos movimientos científicos y disci- plinarios, los cuales han dado origen al florecimiento de un pensamiento unificado alrededor en las ciencias de la complejidad.

Ante este panorama, en la Revista Digital Internacional de Psicología y Ciencia Social se decidió publicar artículos en un amplio rango de campos científicos, puesto que se pretende difundir los avances de la producción científica y tecnológica en los campos de la psicología, las ciencias sociales, de la salud, las ingenierías y las humanidades para fomentar, generar y desarroIlar el conocimiento transdisciplinario entre las distintas disciplinas que comprenden al campo de estudio de las ciencias sociales y de la salud, de las ingenierías y de las humanidades.

Precisamente, el artículo editorial de este número tiene el propósito de guiar y orientar a sus autores y lectores acerca del enfoque y las líneas editoriales que se siguen en la revista. Para lograr este objetivo, en primer término en el artículo editorial se aborda el sentido del concepto de racionalidad, que es el principio que organiza todas las acciones encaminadas a la generación de conocimiento científico; en seguida se examina el papel que tiene la racionalidad en la investigación científica; a continuación se analiza la relación entre racionalidad y verdad; posteriormente se aborda la maneras en que el pensamiento complejo ha cambiado el modo de hacer investigación científica en nuestro tiempo; el artículo finaliza concluyendo que la adopción del enfoque de la complejidad en la investigación científica conlleva un cambio en la mentalidad de la sociedad y se revolucionen las decisiones de poder entre los diferentes niveles de decisión de la comunidad científica.

El artículo de Ramos presenta de una manera clara y concisa el impacto que tienen las intervenciones multidisciplinarias en un centro de desarrollo educativo comunitario que atiende a niños con necesidades educativas especiales.

Los hallazgos mostraron que una atención médica primaria propició la detección y atención temprana de diversos padecimientos y el control del niño sano. El estudio demuestra además cómo el trabajo de múltiples disciplinas tiene efectos positivos, ya que las indicaciones de los optómetras y médicos permitieron un trabajo integral con los niños en el ámbito psicológico.

La autora concluye que el trabajo multidisciplinario impactó favorablemente, puesto que hubo avances en todos los ámbitos de intervención, los niños mostraron una mejoría notable en habilidades sociales, motrices y de autocuidado, gracias al múltiple trabajo de las disciplinas implicadas, cada una de ellas contribuyendo en su ámbito de la salud. 
El siguiente estudio aborda el tema de la autoeficacia que en los últimos años ha tenido un auge importante en la investigación científica, varios de estos estudios se han enfocado a construir instrumentos para medirla. El estudio de Valdez, Jiménez y Villa se centra en evaluar la autoeficacia en el ámbito laboral midiéndola desde una perspectiva contextual, con la finalidad de analizar las propiedades psicométricas del cuestionario de Autoeficacia Profesional (AU-10) en mexicanos que estudian y trabajan.

Los resultados del análisis de confiabilidad y de validez concurrente realizado, mostraron que el cuestionario es confiable y en cuanto a la validez concurrente se encontró una correlación positiva, así como una relación significativamente positiva con las subescalas del Inventario de Desempeño Laboral.

Los autores concluyen que el cuestionario de Autoeficacia Profesional (AU-10) cuenta con adecuadas propiedades psicométricas de confiabilidad y validez, por lo que sugieren que puede utilizarse en investigaciones relacionadas con la autoeficacia en el ámbito laboral en población mexicana.

En el artículo de Remigio y Pérez se reporta un estudio de caso clínico sobre ansiedad y funcionamiento familiar, en el cual se muestra que la influencia de este último puede afectar positiva o negativamente en la salud de los miembros de la familia.

Tomando como base esta idea las autoras idearon su plan terapéutico para el caso clínico bajo la hipótesis de que la relación funcional de la familia se veía influida por la ansiedad que presentaba la madre, y que posiblemente era producto de un conflicto encubierto en el holón conyugal y el síntoma era exteriorizado por los hijos.

Las autoras describen como durante el proceso terapéutico se logró abrir el conflicto que limitaba el funcionamiento familiar, apoyándonos en el enfoque centrado en soluciones y la hipnosis ericksoniana. Además reportan cómo la sujeto después del tiempo de seguimiento continuaba manteniéndose tranquila y su familia también tomando ya decisiones con sus hijos adolescentes.

En el artículo final de este número de la revista, Fabila y Hurtado analizan el problema de la poca atención que recibe en la educación básica el establecimiento de habilidades redactoras, lo cual repercute en las dificultades que muestran la mayoría de los alumnos de educación superior para redactar su tesis. Esta dificultad se convierte en un gran obstáculo al momento del egreso de la licenciatura manifestándose en una incapacidad para elaborar la tesis, ya que al no saber redactar, no hay forma de reflejar el pensamiento, y al tener dificultades para plasmar el pensamiento, se tienen problemas de análisis y reflexión; así como de las habilidades fundamentales para desarrollar cualquier tema académico.

De acuerdo con esto, Fabila y Hurtado plantean la necesidad de identificar el nivel de habilidades de redacción en estudiantes universitarios para observar de qué manera éstas influyen en la elaboración de una tesis de licenciatura. El estudio realizado fue de naturaleza cualitativa realizándose entrevistas estructuradas a 34 alumnos, además a otros 15 alumnos se le requirió que elaborarán un ensayo en el que se les pidió que reflexionarán sobre la manera de transmitir conocimiento.

Los resultados encontrados mostraron que el 93\% de los escritos tenían problemas de ortografía, puntuación, sintaxis y en general mala redacción. Respecto a los entrevistados, el $62 \%$ argumentó que no le gusta hacer investigación, debido a la complejidad de buscar, interpretar y citar la información consultada.

Este segundo número finaliza con dos reseñas de libros, uno es de la autora Analía Verónica Lozada, el otro es de Julio Alfonso Piña López. El primero lleva por título "Familia y Psicología", publicado por la Editorial Dunken en 2015, en él la autora nos habla de la familia, pero no de aquella armoniosa que transmite en la televisión, sino aquella que recibe las complicaciones sociales y que pasa por transiciones, dificultades y fortalezas las cuales marcan su devenir histórico.

Nelida Padilla Gámez hace una reseña amena de este libro y describe las ideas principales que la autora desarrolla a lo largo de su obra, principalmente aqueIlas que tienen que ver con las diversas configuraciones afectivas que hoy conocemos como familia y como la autora toca el tema de las familias reconstruidas, familias homoparentales, las conformadas por dos miembros o las que tienen una mamá o un papá.

El segundo libro de este conjunto de reseñas Ileva por título "Psicología y Salud: Obstáculos y posibilidades para su desarrollo en el siglo XXI", en él su autor examina la manera en que se relaciona la conducta humana con la salud.

En la reseña que hace Sandra Ivonne Muñoz Maldonado del libro motiva a leerlo remarcando una de las muchas bondades que posee relacionadas principalmente con la manera de abordar el papel que tienen los sistemas teóricos en la fundamentación de las explicaciones científicas, así como también en su potencial aplicación práctica en los ámbitos de la prevención y tratamiento de las enfermedades más apremiantes que hoy en día aquejan a amplios sectores. 


\section{Referencias}

Bland, J. M., \& Altman, D. G. (1986). Statistical methods for assessing agreement between two methods of clinical measurement. Lancet, 327(8), 307-310. https://wwwusers.york.ac.uk/ mb55/meas/ba.pdf

Glass, G. V., Wilson, F., \& Gottman, G. (1975). Design and analysis of time-series experiments. Boulder: Colorado Associated Press.
Menard, S. (1991). Longitudinal research. London: SAGE University paper.

Silva-Rodríguez, A. (2004). Diseño de investigación con $\mathrm{N}=1$ o series de tiempo. In A. Silva Rodríguez (Ed.), Métodos cuantitativos en psicología. Un enfoque metodológico (pp. 45-70). México: Trillas.

Yin, R. K. (1984). Case study research: design and methods. Thousand Oaks: Sage Publications, Inc..

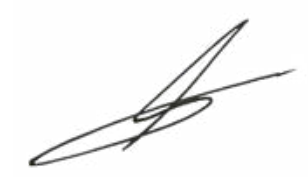

Arturo Silva Rodríguez

Diciembre, 2015 